\title{
Interkulturelles Management - lehren, trainieren, evaluieren. Ein Seminar für interkulturelle Trainer am Institut für Interkulturelle Kommunikation
}

\author{
Olga Rösch
}

„Der Mensch hat dreierlei Wege klug zu handeln: erstens durch Nachdenken - das ist der edelste, zweitens durch Nachahmen, Nachlesen - das ist der leichteste und drittens durch Erfahrung - das ist der bitterste.“

Konfuzius, 5. Jh. v. Chr.

Die alte Weisheit aus dem fernen Osten, stimmig und bedächtig, assoziiert man zunächst kaum mit dem betriebsamen Geschäftsalltag. Der zweite Blick auf das Zitat vergegenwärtigt schon mehr die Aktualität der Sentenz: Denn es geht hier um die kognitiven und affektiven Wege des Lernens und des entsprechenden Handelns. Und damit haben wir in unserem Berufsleben permanent zu tun. Infolge der Internationalisierungs- und Globalisierungsprozesse ist die Zusammenarbeit mit Menschen verschiedener kultureller Prägungen zum Geschäftsalltag geworden. Das bedeutet erst recht ein ständiges und zugleich interkulturelles Lernen. In dieser Gedankenkette ist der fernöstliche Weisheitsspruch im Kontext der interkulturellen Kommunikation heute aktueller denn je. An bitteren Erfahrungen im Geschäftsbereich hat es in der Globalisierungsgeschichte nicht gemangelt. Führungskräfte zahlreicher Unternehmen können darüber berichten.

Aus diesen Erkenntnissen heraus entwickelte sich im Laufe der letzten Jahrzehnte das Bewusstsein für die Notwendigkeit des Wissens um die Fragen der interkulturellen Kommunikation und einer entsprechenden Qualifizierung für Fach- und Führungskräfte. Wir sprechen hier von der interkulturellen Handlungskompetenz als einer überfachlichen Schlüsselqualifikation. Ihr wird im Berufsleben immer mehr Bedeutung beigemessen, und dies aus zahlreichen Gründen:

1. Die Globalisierung der Wirtschaft hat einen dramatischen Wettbewerb ausgelöst.

2. Die internationalen wirtschaftlichen Verflechtungen nehmen weiter zu.

3. Die Qualitätsanforderungen an die internationale Zusammenarbeit in Wirtschaft, Wissenschaft und Politik steigen.

4. Immer mehr internationale Gemeinschaftsunternehmen werden gegründet.

5. Eine große Zahl von ihnen (ca. $70 \%$ ) erfuillt aufgrund mangelnder interkultureller Kompetenz nicht die in sie gesetzten Erwartungen und verursacht zusätzliche Kosten.

6. Die Anzahl multikulturell zusammengesetzter Teams nimmt zu.

7. Der Ausschöpfungsgrad der vorhandenen Leistungspotenziale kann dank interkultureller Kompetenz erhöht werden (z. B. durch Konfliktmanagement).
8. Die hohe Abbrecherquoten von bis zu $50 \%$ der Expatriats müssen vermieden werden.

9. Die Ansprüche ausländischer Kunden an Produktqualität und Serviceleistung steigen.

10. Der kulturäquivalente Umgang mit Mitarbeitern und Kunden bringt einen Wettbewerbsvorteil.

11. Immer mehr Unternehmen beschäftigen immer mehr ausländische Mitarbeiter auf allen Hierarchieebenen im In- und Ausland.

12. Die interkulturelle Zusammenarbeit im eigenen Land wird in quantitativer und qualitativer Hinsicht zunehmen (Stichworte sind: Migration, geplante Zuwanderung, Integration etc.).

13. Europa entwickelt sich zu einer Zuwanderungsregion und Deutschland zu einem der europäischen Zuwanderungszentren [1].

Die neue wirtschaftliche Situation fuihrte dazu, dass sich weltweit neue Forschungsbereiche und Lehrgebiete entwickelten, die unter dem Oberbegriff Interkulturelle Kommunikation mehrere inhaltliche Teilbereiche in sich vereinen. Auch ein neuer Beruf - Interkultureller Trainer - mit einer international organisierten Berufsvereinigung (SIETAR) entstand. SIETAR (Society for Intercultural Education, Training and Research) wurde 1974 in den USA ins Leben gerufen. Aufgrund eines großen Zuspruchs seitens der im Bereich der Interkulturellen Kommunikation international tätigen Fachleute entstanden 1991 auch SIETAR Europa Groups. Dem größten interdisziplinären Global-Netzwerk mit mehr als 3.000 Mitgliedern gehören inzwischen z. B. auch SIETAR Asia Groups an.

1994 wurde SIETAR Deutschland e.V. als ein Teil dieses weltweiten Netzwerkes gegründet. Der Verein bietet ein Forum für alle, die in Berufen mit kulturübergreifenden Aufgabenstellungen tätig sind, und fördert den Austausch zwischen Wissenschaft und Praxis sowie die interdisziplinäre Zusammenarbeit (siehe www.sietardeutschland.de). Seit 1999 sind mehrere SIETAR Regional- und Lokalgruppen als Organisationsstrukturen tätig. Mit Unterstiitzung der Regionalgruppe BerlinBrandenburg der SIETAR Deutschland e.V. werden die Mitglieder aus der Region regelmäßig zu Seminaren für interkulturelle Trainer und anderen Veranstaltungen am Institut für Interkulturelle Kommunikation des TWZ an der TFH Wildau e.V. eingeladen.

Mit dem Seminar „Qualifizierung interkultureller Trainings" im November 2003, dem zweiten in der neuen Veranstaltungsreihe „Kolloquien und Seminare für interkulturelle Trainer" des Instituts für Interkulturelle Kom- 
munikation, wurde die Weiterbildung fuir Trainer („Train the Trainer") erfolgreich fortgesetzt. Zu den 32 Teilnehmern zählten neben den freiberuflich tätigen Trainern auch viele Hochschuldozenten bzw. Wissenschaftler aus mehreren deutschen Universitäten.

Als Referent konnte der renommierte Wissenschaftler Prof. Dr. Alexander Thomas, Leiter der Abteilung fuir Sozial- und Organisationspsychologie an der Universität Regensburg, gewonnen werden. Alexander Thomas ist Autor zahlreicher Publikationen zum Thema interkulturelles Management [2]. Er entwickelte in Deutschland u. a. den Forschungsschwerpunkt kulturvergleichende Psychologie [3] und fuihrt Arbeiten zu Themen wie Psychologie interkulturellen Handelns, Trainings- und Evaluationsforschung, Fragen der interkultureller Handlungskompetenz u. a. durch.

Die Schwerpunkte des Seminars in Wildau waren

- Aktueller Stand der interkulturellen Trainingspraxis,

- Entwicklung und Qualifizierung interkultureller Trainings im Zuge der Globalisierung,

- Diskussion des handlungs- und lerntheoretischen Konzepts interkultureller Kompetenz,

- Trainings als Teil der Kompetenzentwicklung für die Praxis.

Für den Ausbau interkultureller Managementkompetenz sind im Laufe der Jahre je nach Bedarf mehrere Typen von Trainings [4] entwickelt worden. Um ein interkulturelles Training mit einem optimalen Ergebnis für die Zielgruppe durchzufuihren, müssen bei der jeweiligen Konzeption mehrere Aspekte berücksichtigt werden. Vor allem geht es dabei um die Entwicklung der Reflexionsfähigkeit der Teilnehmer in Bezug auf ihre eigene Kultur, um gute Kenntnisse der Kultur des Ziellandes, die Einbeziehung von wissenschaftlichen Erkenntnissen aus der kulturvergleichenden und interkulturellen Forschung, die Berücksichtigung von allgemeinen und spezifischen Anforderungskomplexen an ein effektives Management (z. B. Bewältigung von Konfliktsituationen u. ä.) und - aus diesen Anforderungen heraus - die Festlegung der Trainingsziele. Im Folgenden sind die Bedingungen für interkulturelle Handlungstrainings in einer Übersicht dargestellt.

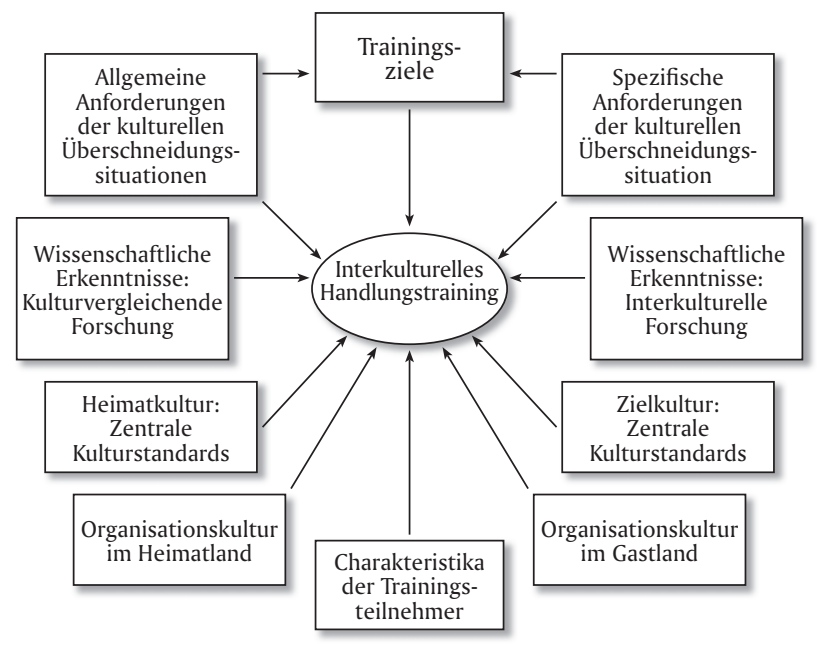

Abbildung nach A. Thomas/K. Hagemann/S. Stumpf, 2003:247 [5]
Die wissenschaftlich fundierten Ausfuihrungen von Alexander Thomas, seine vielfältigen Erfahrungen aus diversen Trainings, ein methodisch-didaktisch durchdachter Aufbau der zu vermittelnden Inhalte, lebhafte Fachdiskussionen und nicht zuletzt die souveräne Art des Referenten machten den Tag für die Teilnehmer aus allen Teilen Deutschlands zu einem Gewinn. Das Seminar „Qualifizierung interkultureller Trainings“ bot viel Stoff zum Nachdenken, es gab aktuelle Literaturtipps zum Weiterlesen, einige Vorgehensweisen, die es wert sind, zum Vorbild genommen zu werden, und vor allem hörte man Erfahrungsberichte, aus denen schon etwas übernommen werden könnte, um sich die eigenen, vielleicht auch „bitteren“ Erfahrungen zu ersparen.

\section{Anmerkungen/Literatur}

[1] In Anlehnung an Seminarunterlagen von Alexander Thomas: „Qualifizierung interkultureller Trainings“, November 2003 an der TFH Wildau.

[2] vgl. z. B. Alexander Thomas/Eva-Ulrike Kinast/Sylvia SchrollMachl (Hrsg.): Handbuch Interkulturelle Kommunikation und Kooperation, Bd. 1 und 2, Göttingen 2003.

[3] vgl. z. B. Alexander Thomas: Psychologie interkulturellen Handelns, Göttingen 1996; A. Thomas: Kulturvergleichende Psychologie - Eine Einführung, Göttingen 1993.

[4] vgl. dazu Olga Rösch: „Personalentwicklung im Kontext der Interkulturellen Kommunikation“, in: Wissenschaftliche Beiträge der TFH Wildau, 2003, S. 78-83.

[5] Alexander Thomas/Katja Hagemann/Siegried Stumpf: „Training interkultureller Kompetenz", in: Niels Bergemann/ Andreas L. J. Sourisseaux (Hrsg.): Interkulturelles Management, Berlin/Heidelberg/New York 2003, S. 237-272.

\section{Autorin}

Prof. Dr. Olga Rösch

Technische Fachhochschule Wildau

Fachbereich Ingenieurwesen/Wirtschaftsingenieurwesen Leiterin des Instituts fuir Interkulturelle Kommunikation des TWZ an der TFH Wildau e. V.

Tel. +493375 508-367

E-mail: roesch@sprz.tfh-wildau.de 